\title{
Proyectos STEAM con LEGO Mindstorms para educación primaria en España
}

\author{
F.Ruiz $^{\text {a }}$, A.Zapatera ${ }^{\text {b }}$, N.Montés ${ }^{\text {c }}$, N.Rosillo ${ }^{\mathrm{d}}$ \\ Universidad Cardenal Herrera CEU, España, aitn_faruiz@fomento.edu ${ }^{\text {a }}$, \\ alberto.zapatera@uchceu.es ${ }^{\mathrm{b}}$,nicolas.montes@uchceu.es ${ }^{\mathrm{c}}$,nrosillo@uchceu.es ${ }^{\mathrm{d}}$.
}

\section{Resumen}

En el presente trabajo, se analiza cómo utilizar LEGO ${ }^{\circledR}$ MINDSTORMS ${ }^{\circledR}$ Education EV3 para proyectos STEAM enmarcados en el curriculum de educación de la ley LOMCE 2013.

En nuestros trabajos anteriores se desarrolla e implementa una intervención propuesta a través de un proyecto de aprendizaje STEAM obtenido del análisis de la LOMCE y que utiliza la robótica educativa como una herramienta de enseñanza que incorpora diferentes elementos metodológicos como el aula inversa, y el aprendizaje basado en proyectos, así como el aprendizaje cooperativo.

También en nuestros trabajos anteriores se ha desarrollado una nueva plataforma educativa basada en el paquete Matlab Simulink para la enseñanza de robótica utilizando la plataforma Lego EV3. Esta plataforma permite desarrollar un entorno en tiempo real para enseñar inicialmente temas de robótica, programación y en general, temas de ingeniería. Por ello es utilizada actualmente en proyectos STEM, sin embargo, el uso de piezas LEGO le otorga la versatilidad de introducir la A de Arte.

El presente artículo analiza como introducir la A a partir del uso de LEGO Mindstorm, lo que nos otorga una herramienta ideal para su uso en proyectos STEAM.

Palabras clave: LOMCE, STEAM, LEGO Mindstorm, Robótica, EV3. 


\section{Introducción}

Los cambios sociales y tecnológicos del siglo XXI plantean la necesidad de redefinir el modelo de enseñanza, en especial los aspectos relacionados con la capacidad de utilizar adecuadamente los conocimientos científicos y tecnológicos para tomar decisiones de forma responsable, autónoma y crítica sobre el mundo natural y sus cambios.

En respuesta a este desafío, primero en Estados Unidos y después en Europa, surgió la propuesta STEM como una estrategia para la educación en ciencia (S), tecnología (T), ingeniería (E) y matemáticas (M), enfocada al desarrollo de competencias y habilidades tecnológicas. La propuesta STEM, basada en la resolución de problemas y situaciones abiertas y no estructuradas de la vida cotidiana, pretende desarrollar un enfoque interdisciplinario del proceso de enseñanza y aprendizaje utilizando herramientas tecnológicas.

En el aprendizaje STEM el alumno construye su propio conocimiento, por lo que puede considerarse un aprendizaje constructivista que utiliza metodologías activas como el aprendizaje basado en proyectos, la enseñanza para la comprensión, aprendizaje basado en problemas, el aprendizaje cooperativo, el aula inversa o el construccionismo.

Desde esta perspectiva, uno de los ámbitos de aplicación más eficaces en el contexto del aprendizaje STEM es la robótica educativa, que propone construir dispositivos tecnológicos que permitan a los alumnos programarlos para realizar determinadas acciones e interactuar con ellos. Dentro de la robótica educativa, se ha generalizado el uso de las plataformas de bajo coste, las más comunes son (Irigoyen,, Larzabal \& Priego, 2013): Arduino, la Raspberry Pi, Kinect, etc. En el caso de los robot móviles, en los últimos años ha sido posible adquirir robots móviles de bajo coste como Adept (ADEPT, 2014), Moway (Moway, 2017), epuck (Epuck, 2014) y LEGO Mindstorms (Mindstorm LEGO, 2017).

La robótica educativa se ha convertido en un recurso didáctico innovador que permite al alumno construir conocimiento, que mejora la motivación, el interés y el rendimiento en las materias STEM y desarrolla habilidades sociales, el trabajo cooperativo, la creatividad, etc.

Aunque todas estas plataformas son útiles, el rol que LEGO Mindstorm ha jugado en la educación en los últimos años es más que notable. La principal razón es que el kit de LEGO Mindstorm contiene software y hardware para crear prototipos de una amplia variedad. Incluye el Brick, sensores, motores y partes de la línea Technic para crear sistemas mecánicos. Este kit también se puede combinar con cualquier kit de la marca LEGO, multiplicando su capacidad. La robótica de Lego es fundamentalmente una herramienta constructivista, con estudiantes aprovechando su conocimiento y experiencia para resolver un problema del mundo real y de manera consistente cuestionar y desafiar ese conocimiento a medida que se desarrollan. 


\section{Objetivos}

En los últimos años, los kits de LEGO se vienen usando de forma generalizada para el aprendizaje STEM, (STEM-LEGO, s.f.). Sin embargo, no se han desarrollado todavía proyectos STEAM, incluyendo la A de Arte.

El objetivo de este artículo es utilizar el análisis del currículo de $4^{\circ}, 5^{\circ}$ y $6^{\circ}$ de Educación Primaria (LOMCE, 2013) en el ámbito de la Comunidad Valenciana (D108/2014, DOCV, 2014) desarrollado en nuestros trabajos anteriores, ver (Ruiz, Zapatera, \& Montes Sanchez, s.f.) para determinar cómo utilizar LEGO en la A de las áreas de oportunidad detectadas.

\section{Antecedentes}

\subsection{Análisis curricular y robótica educativa}

El aprendizaje STEM es un objetivo fundamental de las agendas educativas de numerosos países y organismos internacionales, por lo que numerosos estudios (Yackman \& Lee, 2012) (Yakman, 2008) han comenzado a centrarse en la problemática de su aprendizaje, investigando casos prácticos particulares y teorizando sobre las relaciones que debían establecerse entre las cuatro disciplinas.

Al revisar el concepto de aprendizaje STEM, (Yakman, 2008) inició el aprendizaje STEAM al incluir el arte como agente integrador de las cuatro disciplinas. El plan educativo de Corea se fundamenta en el marco teórico de Yakman (2008) que considera el arte en una acepción muy amplia que abarca campos como el lenguaje, las ciencias sociales o las artes físicas, además de las tradicionalmente consideradas como las bellas artes. Otro referente mundial del aprendizaje STEAM, la Rode Island Design School (RIDS), incorpora también el arte, resaltando aspectos como la estética, el diseño y la imaginación.

De esta forma. el aprendizaje STEAM y la robótica educativa intentan unir a la adquisición de competencias científico-técnicas, el desarrollo de la creatividad y la innovación, generando situaciones de aprendizaje más creativas y motivadoras, aumentando el interés de los alumnos y desarrollando sus habilidades creativas, de resolución de problemas y de cooperación.

Aunque en la literatura científica pueden encontrase diferentes proyectos de robótica educativa que incluyen la tecnología lego en el contexto del aprendizaje STEM, es necesario investigar el desarrollo de estos proyectos en el contexto STEAM, en concreto, la participación del arte en los proyectos STEAM que utilizan tecnología lego. 
Para responder a las preguntas de investigación se analizó el currículo de $4^{\circ}, 5^{\circ}$ y $6^{\circ}$ de Educación Primaria (LOMCE) en el ámbito de la Comunidad Valenciana (D108/2014, DOCV, 2014) para determinar las áreas de oportunidad de proyectos de aprendizaje STEAM.

Un tema o materia es un área de oportunidad de un proyecto de aprendizaje STEAM si cumple las características del reto o pregunta desafiante del aprendizaje basado en proyectos (Larmer, Mergendoller, \& Boss, 2015) y las características del tópico generativo de la enseñanza por comprensión (Del Pozo, 2009).

Para determinar las áreas de oportunidad se partió de la hipótesis de que las disciplinas STEAM aparecen en el currículum LOMCE en las asignaturas de Ciencias de la Naturaleza, Ciencias Sociales, Matemáticas y Educación Plástica, por lo que en (Ruiz, Zapatera,\& Montes, s.f.) se analizaron los contenidos a partir de los ítems curriculares de las cuatro asignaturas en los cursos $4^{\circ}, 5^{\circ}$ y $6^{\circ}$ de Educación Primaria.

En una primera etapa se localizaron los 1020 ítems curriculares de las asignaturas y cursos seleccionados, se eliminaron los ítems redundantes que aparecían exactamente iguales en dos o más cursos, y se agruparon los ítems con contenidos semejantes en 281 grupos curriculares. En una segunda etapa se seleccionaron los 218 que tenían contenidos STEAM, se clasificaron dentro de las disciplinas STEAM y se agruparon en 41 áreas temáticas que contenían grupos curriculares relacionados entre sí. En una tercera etapa se establecieron relaciones entre las áreas temáticas de cada disciplina y se seleccionaron las 21 áreas de oportunidad que eran las que más relaciones tenían. Tanto las áreas temáticas como en las de oportunidad, se diferenciaron entre conceptuales y no conceptuales, que desarrollan contenidos procedimentales o actitudinales (Tabla 1).

\begin{tabular}{lccccccccccc}
\hline & \multicolumn{4}{c}{ Grupos curriculares } & \multicolumn{3}{c}{ Áreas temáticas } & \multicolumn{3}{c}{ Áreas de oportunidad } \\
\hline STEAM & Mat. & C. N. C. S. E. P. Total & C. & No C. Total & \multicolumn{2}{c}{ C. } & No C. Total \\
\hline S (Ciencias) & 0 & 29 & 23 & 0 & 52 & 9 & 1 & 10 & 4 & 1 & 5 \\
T (Tecnología) & 2 & 13 & 4 & 3 & 22 & 1 & 3 & 4 & 1 & 2 & 3 \\
E (Ingeniería) & 34 & 11 & 3 & 9 & 57 & 7 & 3 & 10 & 2 & 3 & 5 \\
A (Arte) & 1 & 1 & 1 & 35 & 38 & 4 & 2 & 6 & 2 & 2 & 4 \\
M (Matemáticas) & 48 & 0 & 1 & 0 & 49 & 9 & 2 & 11 & 2 & 2 & 4 \\
\hline Total & 85 & 54 & 32 & 47 & 218 & 30 & 11 & 41 & 11 & 10 & 21 \\
\hline
\end{tabular}

Tabla 1. Clasificación STEAM de grupos curriculares, áreas temáticas y áreas de oportunidad. 


\subsection{Lego Mindstorm como plataforma educativa}

En nuestros trabajos anteriores se propuso una nueva plataforma basada en LEGO Mindstorms como plataforma educativa, (Montés, Rosillo, Hilario, \& Mora, 2018). En particular, esta nueva herramienta se basaba en el último modelo de LEGO Mindstrom, EV3 y su conexión con Matlab/Simulink, para así conseguir una herramienta en tiempo real que permita la interactuación entre el prototipo diseñado con LEGO Mindstorm y el alumno. Con esta herramienta se pueden desarrollar aplicaciones interactivas en tiempo real como la mostrada en la figura 1, ver (Del Pozo, 2009). En este ejemplo, el alumno puede modificar el destino del robot (punto rojo) con la mano en tiempo real.
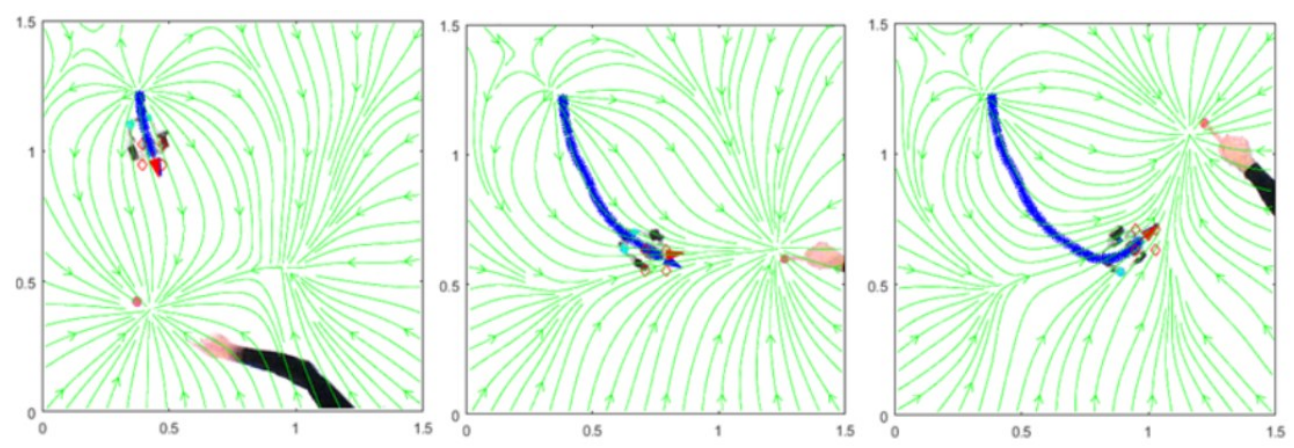

Fig. 1 Ejemplo de aplicación de la herramienta Matlab-Simulink-Ev3

\section{Análisis del Arte para educación primaria en España}

El diseño de un proyecto de aprendizaje STEAM debe partir de un área de oportunidad conceptual principal, para luego, ir seleccionando otras áreas conectadas con ella y así estudiar la participación del arte en proyectos de robótica educativa en el contexto STEAM. Las seis áreas temáticas de la disciplina del arte localizadas en el diagrama general son las mostradas en la siguiente figura. 


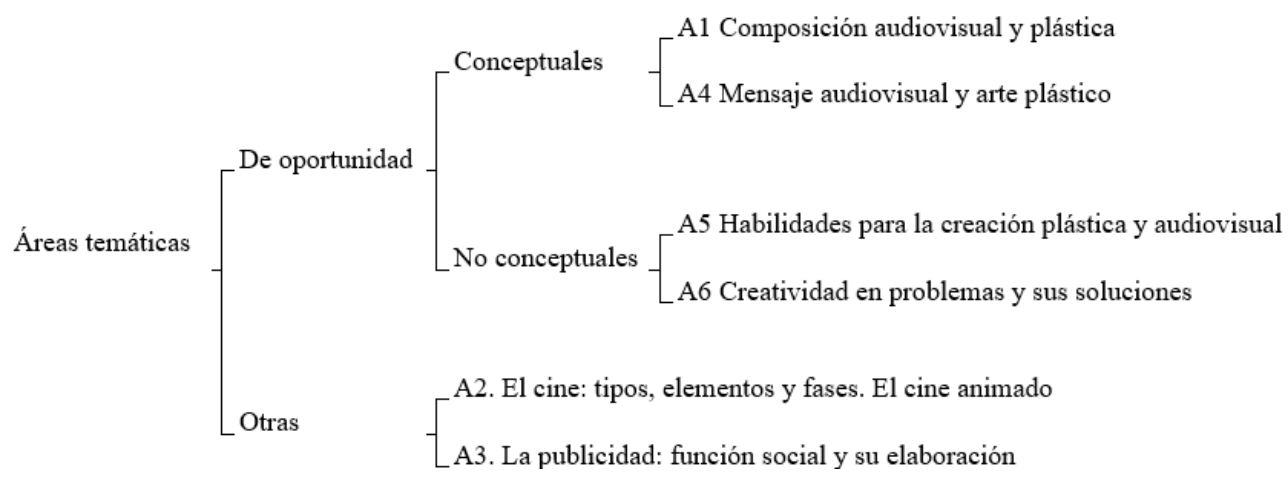

Fig. 2. Áreas temáticas de la disciplina arte.

Las dos áreas de oportunidad conceptuales, "Composición audiovisual y plástica" (A1) y "Mensaje audiovisual y el arte plástico" (A4) como área de oportunidad conceptual principal comprende 10 grupos curriculares de los tres cursos de la asignatura de Educación Plástica (EP) y que cubren 34 ítems no redundantes y 47 ítems curriculares, ver tabla 2.

\begin{tabular}{|c|c|c|}
\hline Asignatura & Grupo curricular & Área de oportunidad \\
\hline EP-456 & Alfabetización audiovisual & \multirow{10}{*}{$\begin{array}{l}\text { COMPOSICIÓN } \\
\text { AUDIOVISUAL Y } \\
\text { PLÁSTICA }\end{array}$} \\
\hline EP-456 & Expresividad de los elementos visuales & \\
\hline EP-5 & Formas y volúmenes formados por planos & \\
\hline EP-456 & El color, tipos, propiedades, simbología... & \\
\hline EP-45 & La luz, la sombra y el volumen & \\
\hline EP-456 & Trabajo con texturas & \\
\hline EP-5 & Mosaicos, cenefas y polígonos estrellados & \\
\hline EP-456 & Elementos de composición & \\
\hline EP-456 & Técnicas plásticas bi y tridimensionales & \\
\hline EP-456 & Materiales de soporte & \\
\hline
\end{tabular}

La segunda área de oportunidad "El mensaje audiovisual y el arte plástico" (A4) comprende 6 grupos curriculares de los tres cursos de la asignatura Educación Plástica (EP), lo que suponen 17 ítems no redundantes y 25 ítems curriculares, Tabla 3. 


\begin{tabular}{|c|c|c|}
\hline Asignatura & Grupo curricular & Área de oportunidad \\
\hline EP-4 & Identificación, análisis y función social & \multirow{6}{*}{$\begin{array}{l}\text { EL MENSAJE } \\
\text { AUDIOVISUAL Y EL } \\
\text { ARTE PLÁSTICO }\end{array}$} \\
\hline EP-456 & Comprensión del mensaje audiovisual & \\
\hline EP-6 & Dominio del lenguaje audiovisual & \\
\hline EP-456 & Vocabulario y dominio de la terminología & \\
\hline EP-456 & Identificación de obras, respeto y valoración & \\
\hline EP-456 & Interés por el arte plástico; museos, cine... & \\
\hline
\end{tabular}

Las conexiones intradisciplinares e interdisciplinares del área de oportunidad "El mensaje audiovisual y el arte plástico" (A4) son las mismas que las del área de oportunidad "Composición audiovisual y plástica" (A1) por lo que los diagramas y las áreas temáticas conectadas coinciden (Figura 3) (Tabla 3).

De esta manera, dentro de la disciplina del arte se han localizado dos áreas de oportunidad, "Composición audiovisual y plástica" (A1) y "El mensaje audiovisual y el arte plástico" (A4), en torno a las que se pueden desarrollar proyectos STEAM con robótica educativa en el entorno LEGO. Sin embargo, la mayor aportación del arte a este tipo de proyecto la realizan las áreas de oportunidad no conceptuales, o áreas de oportunidad procedimentales y actitudinales "Habilidad para la creación plástica y audiovisual" (A5) y "Creatividad en problemas y sus soluciones" (A6).

\section{LEGO Mindstorm en la A de STEAM en educación primaria}

En la última década, el uso de piezas LEGO para crear arte se ha puesto cada vez más de moda. Existen artistas que se han especializado en crear obras artísticas con piezas de LEGO. Se les conoce como "BrickArtists". Uno de los más representativos es Nathan Sawaya. Él es un artista americano que construye esculturas 3D y mosaicos de gran tamaño con piezas de LEGO, (Sawaya, s.f.). Su trabajo se puede visitar en las galerías neoyorquinas, Avant Gallery, Agora Gallery, Artsicle and Discovery Times Square in New York City. Otro de los “BrickArtist” más representativos es Jefrey Kong, (Kong, s.f.). Sean Keney, (Keney, s.f.), Kevin Hall, (Hall, s.f.), etc. Por lo tanto, el uso de bricks de LEGO ya proporciona al docente la herramienta necesaria para, a partir del uso de estas piezas poder introducir la A de STEAM dentro de sus proyectos. En los presentes subapartados se presentan como se podría integrar para cada una de las áreas temáticas de la disciplina Arte extraídas de la LOMCE. 


\subsection{Composición audiovisual y plástica.}

Como podemos ver en la Tabla 2, en esta área temática los alumnos deben de trabajar formas y volúmenes, colores, tipos propiedades, simbología, la luz, mosaicos, cenefas, elementos de composición, técnicas plásticas bi y tridimensionales, etc. Sin lugar a dudas, todos estos grupos curriculares se pueden desarrollar con piezas LEGO.

\subsection{El cine: Tipos, elementos y fases. El cine animado.}

Quizás este grupo curricular sea el que a priori resulte más difícil para su uso con piezas LEGO, sin embargo, también lo es para los docentes que no las usan. Actualmente, los profesores se apoyan en recursos didácticos donde uno de los más usados es la construcción y fabricación de un cine. En ese caso, el uso de Piezas LEGO para la construcción de escenarios y escenas animadas tipo Stop Motion permitiría su inclusión.

\subsection{La publicidad. Función social y su elaboración.}

De la misma manera que en el apartado anterior, cuando los docentes se enfrentan a enseñar este tipo de materias en el aula, suelen utilizar distintos tipos de recursos. En este caso, el uso de piezas LEGO para el desarrollo de anuncios como material de apoyo serviría.

\subsection{Mensaje audiovisual y arte plástico.}

La existencia de los "BrickArtists" y sus obras de arte ya dan pie a trabajar esta área desde LEGO ya que como se refleja en la Tabla 3 incluye aspectos como la identificación de obras, respeto y valoración o el interés por el arte plástico; museos, cine, etc.

Por otro lado, uno de los recursos más utilizados por los docentes suele ser la interpretación de obras de arte por parte del alumnado. Realizar estas interpretaciones con piezas LEGO es una de las alternativas.

\subsection{Habilidades para la creación plástica y audiovisual.}

Esta área temática hace referencia a la habilidad del estudiante para crear sus propias composiciones plásticas y audiovisuales. Cualquiera de los usos de las piezas LEGO descritos en los apartados anteriores también daría pie a evaluar la habilidad para la creación plástica.

\subsection{Creatividad en problemas y sus soluciones.}

Igual que en el apartado anterior, el mero uso de las piezas LEGO para resolver cualquier ejercicio plástico hace que a la vez se pueda valorar el grado de creatividad mostrado para resolver la situación. 


\section{Conclusiones}

En el presente trabajo, se analiza cómo utilizar LEGO ${ }^{\circledR}$ MINDSTORMS ${ }^{\circledR}$ Education EV3 para proyectos STEAM enmarcados en el curriculum de educación de la ley LOMCE 2013.

En nuestros trabajos anteriores se desarrolló e implemento una intervención propuesta a través de un proyecto de aprendizaje STEAM obtenido del análisis de la LOMCE y que utiliza la robótica educativa como una herramienta de enseñanza que incorpora diferentes elementos metodológicos como el aula inversa, y el aprendizaje basado en proyectos, así como el aprendizaje cooperativo.

También en nuestros trabajos anteriores se ha desarrollado una nueva plataforma educativa basada en el paquete Matlab Simulink para la enseñanza de robótica utilizando la plataforma Lego EV3. Esta plataforma permite desarrollar un entorno en tiempo real para enseñar inicialmente temas de robótica, programación y en general, temas de ingeniería. Por ello es utilizada actualmente en proyectos STEM Sin embargo, el uso de piezas LEGO le otorga la versatilidad de introducir la A de Arte.

El presente artículo analiza como introducir la A a partir del uso de LEGO Mindstorm, lo que nos otorga una herramienta ideal para su uso en proyectos STEAM. La A de Arte se introduce de manera directa ya que existe un movimiento internacional conocido como los "BrickArtists". Estos artistas crean arte a partir de piezas LEGO. Por lo tanto, no es difícil introducir en la A de Arte de los proyectos STEAM diseñados piezas LEGO.

\section{Referencias}

ADEPT. (2014). Adept Mobile Robots. Obtenido de http://activrobots.com

Danahy, E., Wang, E., Brockman, J., CArberry, A., \& Shapiro, B. (2014). LEGO-based robotics in Higher Education: 15 years of students creativity. International Journal of Advanced Robotics Systems, vol 11, Is 2, pp 157-172.

Del Pozo, M. (2009). Aprendizaje Inteligente. Educación secundaria en el colegio Montserrat. Badalona. Teckman Books. Obtenido de https://www.youtube.com/watch?v=LC_kFZPmOH0\&t=249s

Epuck. (2014). e-puck education robot. Obtenido de http://www.e-puck.org

Hall, K. (s.f.). Obtenido de https://www.linkedin.com/in/kevin-hall-90678a53/ 
Irigoyen, E., Larzabal, E. \& Priego, R. (2013). Low-cost platforms used in Control Education: An educational case Study. 10th IFAC Symposium Advances in Control Education, 256-261.

Keney, S. (s.f.). Obtenido de http://www.seankenney.com/

Kong, J. (s.f.). Jeffrey Kong. Obtenido de https://artisanbricks.com

Larmer, J., Mergendoller, J., \& Boss, S. (2015). Setting the standard for project based learning. En $A S C D$.

LOE. (2006). Obtenido de https:/www.boe.es/buscar/doc.php?id=BOE-A-2006-7899, visitada el 30/11/2017.

LOMCE. (2013). Obtenido de https://www.boe.es/buscar/act.php?id=BOE-A-2013-12886, visitada el 30/11/2017.

Mindstorm LEGO. (2017). LEGO Mindstorm. Obtenido de http://mindstorms.lego.com

Montés Sánchez, N., Rosillo Guerrero, N., Hilario Perez, L., \& Mora Aguilar, M. (2018). A novel educatinal platform based on Matlab/Simulink/LEGO EV3 for teaching with robots. 12th International Technology, Education and Development Conference. Valencia.

Moway. (2017). Moway Robots. Obtenido de http://moway-robot.com

Ruiz, F., Zapatera, A., \& Montes Sanchez, N. (s.f.). Diseño y puesta en marcha de proyectos STEAM para el sistema educativo español en educación primaria. Revista en Educación. En revisión.

Sawaya, N. (s.f.). TheBrickArtist. Obtenido de www.brickartist.com

STEM-LEGO. (s.f.). Obtenido de https://thestemlaboratory.com/lego-stem-activities/

Yackman, G., \& Lee, Y. (2012). Exploring the exemplary STEAM education in the US as a practical educational framework for Korea. Journal of Korea Association Science Education, 32(6); 1072-1086.

Yakman, G. (2008). STEAM Education: an overview of creating a model of integrative education. En Pupils Attitudes TowardsTechnology (PATT) (págs. 35-358). 